\title{
Humor gráfico y comprensión de deseos*
}

\section{Graphic Humor and Understanding of Desires}

\section{Mónica Roncancio Moreno** \\ Rebeca Puche-Navarro-Navarro \\ Universidad del Valle, Colombia}

Recibido: 2 de diciembre de 2011 Revisado: 4 de marzo de 2012

Aceptado: 30 de mayo de 2012

\section{Resumen}

El presente artículo tiene como propósito documentar la comprensión de los deseos a partir de dos aproximaciones: situaciones que utilizan el humor gráfico y situaciones clásicas de preferencias con niños entre 2.8 y 3 años. Se trabaja con el método microgenético combinado con un estudio en series de tiempo durante 10 sesiones de aplicación (dos meses y medio). Se realizan dos tipos de análisis, uno intragrupo y uno intraindividual que permite capturar los diversos caminos por los cuales transitan los niños al enfrentarse a las tareas. Los resultados arrojan la identificación de tres tendencias en los desempeños: estable, descendente y ascendente. Igualmente, se pone en evidencia la importancia de trabajar otras vías para el estudio de los procesos cognitivos en términos metodológicos y se confirma el humor gráfico como alternativa para el estudio de los estados mentales.

Palabras clave: Humor, incongruencia, teoría de la mente, preferencias, comprensión de deseos. 


\section{Abstract}

This paper aims to document the understanding of desires from two approaches, one is graphic humor and the other is preferences with children between two years eight months and three years. Methodologically, we work with the microgenetic method combined with a time-series study over 10 sessions for two months. Two types of analysis are used, an intra-group and through an intrasubject one; the latter captures the various ways which children go when faced with tasks. The results identify three trends in performance: stable trend, downward trend, and upward trend. They also show the importance of working on other ways for the study of cognitive processes in terms of methodology and these results confirm graphic humor as an alternative for the study of mental states.

Keywords: Humor, incongruence, theory of mind, preferences, understanding of desires.

Desde la década de los ochenta, el estudio de la comprensión de estados mentales o Teoría de la Mente (TdM) se integró al estudio de la psicología del desarrollo como disciplina. Objetos que antes eran considerados del campo de una 'folk psychology', como las creencias, los deseos y las intenciones fueron asumidos y la psicología pasó a ocuparse de los estados mentales (Astington, 1998; Benavides, 2010; Benavides \& Roncancio, 2009, 2011; Puche-Navarro \& Lozano, 2002; Arbeláez, Salgado-Montejo \& Velasco, 2010). Con el paso del tiempo los estudios en este campo perfilaron toda su potencia hasta mostrarse como uno de los más fértiles en psicología. Hoy en día sus relaciones con el desarrollo cognitivo son privilegiadas y de más en más complejas (para una revisión ver Cadinu \& Kiesner, 2000; Doherty, 2009; Taylor, 1996).

Una de las tendencias en los estudios de la TdM que se puede identificar en la bibliografía reciente (Reddy, 2006, 2008; Reddy \& Morris, 2004) y menos reciente (Bloom \& German, 2000; Leslie \& Polizzi, 1998) ha sido una preocupación metodológica. A pesar de la dominancia de la Falsa Creencia (FC) como dispositivo en el estudio de la atribución de las creencias y deseos en el otro, se registra una búsqueda de nuevas estrategias que permitan conocer el desarrollo de la capacidad de atribuir estados mentales a otros. Esa búsqueda metodológica ha sido relativamente diversa pues va desde -variaciones con el método microgenético- (Amsterlaw \& Wellman, 2006; Flynn, 2006; Flynn, O’Malley \& Wood, 2004), pasando por metodologías provenientes de la neurociencia (Liu,
Melzoft \& Wellman, 2009), situaciones que toman como base el juego de simulación (Dias, Roazzi, O’Brien \& Harris, 2005) hasta la utilización del humor como puerta de entrada a la teoría de la mente (Puche-Navarro, 2004, 2009, 2012; PucheNavarro \& Lozano, 2002).

El objeto sobre el cual gravita el presente estudio es doble. Por una parte la comprensión de los deseos. Para ello utiliza el método microgenético combinado con las series de tiempo, e intenta establecer el puente entre humor visual y deseo mediante la comprensión del humor gráfico (Puche-Navarro, 2004, 2009, 2012). Por otra parte, enriquece la evidencia de que dicha clase de humor es un vehículo que permite capturar información preciosa sobre lo precoz y sorprendente que resulta la emergencia de las condiciones mentalistas del sujeto en el caso de los deseos.

\section{El estudio del deseo en la teoría de la mente}

Es bien conocido que la temática principal para la teoría de la mente ha sido el estudio de las creencias (Astington, 1998; Astington \& Barriault, 2001; Astington, Harris \& Olson, 1988; BaronCohen, Leslie \& Frith, 1985; Gopnik, 1993; Gopnik \& Astington, 1988; Wellman, 1990; Wimmer \& Perner, 1983). Sin embargo, la comprensión de los deseos resulta también particularmente atractiva. La razón del papel que tiene la compresión de los deseos en la TdM deriva tal vez de su temprana aparición (Astington, 1998; Bartsch \& Wellman, 
1995; Gopnik, 1993; Gopnik \& Astington, 1988; Repacholi \& Gopnik, 1997; Wimmer \& Perner, 1983; Woolley \& Wellman, 1993). Algunos estudios relacionan esta comprensión temprana con la aparición de los deseos básicos, tales como los que llevan a la satisfacción de necesidades fisiológicas: hambre, sueño, entre otras (Astington, 1998) o incluso con el empleo de términos mentalistas que tienen que ver con deseos desde los primeros años de vida (Bartsch \& Wellman, 1995).

En la década del ochenta, la bibliografía en TdM que se ocupa de la comprensión de deseos no es muy amplia. A finales de los años ochenta e inicios de los noventa, aparecen los primeros acercamientos al estudio de la comprensión de los deseos simples en otros (Bartsch \& Wellman, 1989; Wellman \& Banerjee, 1991; Wellman \& Woolley, 1990). Las tareas que usualmente se empleaban eran de búsqueda de objetos y de preferencias. Más adelante, hacia finales de los noventa y comienzos del 2000 aparece el interés por la comprensión de los funcionamientos inferenciales y deseos conflictivos (Cassidy et ál., 2005; Leyva, 2003; Rakoczy, Warneken \& Tomasello, 2007; Repacholi \& Gopnik, 1997). Metodológicamente, para abordarlos, se emplearon dos tipos de indicios: tareas de claves atencionales (Eskritt \& Lee, 2007; Flom, Lee \& Muir, 2007; Lee, Eskritt, Symons \& Muir, 1998; Montgomery, Bach \& Moran, 1998) y tareas de preferencias (Cassidy, 1998; Cassidy et ál., 2005; Flavell, J., Flavell, E., Green \& Moses, 1990; Moore, Jarrold, Russell, Lumb \& Sapp, 1995; Repacholi \& Gopnik, 1998; Rieffe, Meerum Terwogt, Koops, Stegge \& Oomen, 2001). Recientemente, se ha incrementado el número de investigaciones que estudian los deseos (Brandone \& Wellman, 2009; Cassidy et ál., 2005; Ferres, 2003; Hughes, Lecce \& Wilson, 2007; Liu, Meltzoff \& Wellman, 2009; Rakoczy et ál., 2007; Terwogt \& Rieffe, 2004; Wertz \& German, 2007). Entre las tendencias más representativas se encuentra el interés por el estudio de la relación entre deseosmetas insatisfechas (Brandone \& Wellman, 2009) y por las bases neurales y los mecanismos que subyacen a su comprensión (Liu et ál., 2009) (para una revisión más detallada ver Roncancio, 2010).
En ese contexto y durante los casi 20 años que se ha investigado la comprensión de los deseos se generaron, como era de esperarse, diferentes tareas; sin embargo, no existe una situación estándar como en el caso de la situación de las falsas creencias. Las situaciones han variado de acuerdo con el tópico sobre el cual se enfatiza el estudio de los deseos, ya sean simples, conflictivos (cuando dos personas tienen deseos diferentes frente al mismo objeto) o traten de dar cuenta de los funcionamientos inferenciales.

En este estudio, se explora la manera cómo el niño comprende un estado mental definido por el deseo, por medio de situaciones humorísticas graficas. Se establece el puente bosquejado entre los estados mentales y el humor, relación explorada en investigaciones previas (Puche-Navarro, 2004, 2009, 2012). De esta forma se tratará de confirmar la viabilidad de usar una situación de humor gráfico para inferir deseos, tomando como plataforma el método microgenético y de avanzar en la información que aún falta acerca de la comprensión de los deseos en los niños.

\section{El estudio del humor gráfico y su relación con la comprensión de estados mentales}

El estudio del humor ha sido considerado, tanto conceptual como metodológicamente, un campo novedoso en psicología cognitiva (Bariaud, 1983; McGhee, 1979, Puche-Navarro, 2004, 2009, 2012; Reddy, 2008). Comprender un chiste o una broma, implica habilidades cognitivas y sociales que van de la mano de complejas representaciones cognitivas. Existe una cierta bibliografía en este campo, no obstante, ella no ha sido dominante. La cuestión es que estos estudios sobre el humor han abierto algunas líneas en el campo del funcionamiento de la actividad cognitiva allí involucrada (Hoicka \& Gattis, 2008; J ohnson \& Mervis, 1997; Loizou, 2005, 2006; Lozano \& Puche-Navarro, 1998; Puche-Navarro, 2004; Puche-Navarro \& Lozano, 2002; Reddy, 1991, 2001, 2008; Strick, Holland, van Baaren \& van Knippenberg, 2009). El International J ournal of Humor Research publica desde 1988 y presenta un variado repertorio de 
artículos tanto empíricos como teóricos. Lo cierto es que el conjunto de la bibliografía sobre el humor cubre temáticas muy diversas que abordan aspectos clínicos, interculturales, afectivos y cognitivos. Asimismo, los investigadores han estudiado el humor y su relación con el lenguaje (J honson \& Mervis, 1997), el humor y su relación con explicaciones verbales (Loizou, 2005, 2006); la producción del humor en el bebé y el infante (Reddy, 1991, 2001, 2008), el humor y su relación con las emociones (Strick et ál., 2009) y de manera más específica la comprensión del humor gráfico y los estados mentales (Puche-Navarro, 2004, 2009, 2012; Puche-Navarro \& Lozano, 2002).

Específicamente, para la presente investigación se ahondará en el humor gráfico y dentro de este una clase particular corresponde a los chistes gráficos 'mentalistas' (Puche-Navarro, 2004, 2009, 2012). Los chistes mentalistas involucran, como su nombre lo deja ver, contradicciones relacionadas con el estado mental de un personaje y "propicia la temprana capacidad de los niños a interpretar y reproducir en su propio sistema emocional, los estados, intenciones, creencias o suposiciones identificadas en el objeto visual" (Puche-Navarro, 2012, p. 12).

De cara a mostrar lo fértil que puede resultar el escenario del humor para estudiar los estados mentales y de manera específica los deseos, es necesario entender que la base sobre la cual se construye cualquier tipo de humor (gráfico o verbal) es la incongruencia. La incongruencia supone remitirse "a una situación en la que la comprensión de una relación previsible, estructural o secuencial (espacial o temporalmente entendida) es esperada, y en cambio ocurre algo inesperado" (Puche-Navarro \& Lozano, 2002, p. 32) (McGhee, 1988; Puche-Navarro, 2004; Reddy, 2008). La sorpresa que genera el chiste en el niño, permite la comprensión de la violación y subversión de lo real (Puche-Navarro, 2004, 2009; Puche-Navarro \& Lozano, 2002).

El deseo, la creencia o el sentimiento se encuentran entonces implícitos en la relación incongruente a manera de proyección del estado mental caracterizado en el chiste mentalista, así como para estudiar los procesos inferenciales allí involucrados (Puche-Navarro, 2009; Combariza \& Puche-Navarro, 2009). Con este tipo de abordaje se trabaja la comprensión de los estados mentales y su relación con los funcionamientos inferenciales del sujeto. En los chistes de tipo mentalista se debe inferir la relación transgredida de la proyección del estado mental de un personaje. Por ejemplo, teóricamente, Puche-Navarro y Lozano (2002) al referirse al chiste mentalista aluden a Dennett para plantearlo como sistema intencional, afirman: "la naturaleza de la incongruencia en los chistes mentalistas funciona como un sistema intencional de segundo orden" (p. 344) y, según Astington (1998), "un sistema intencional de segundo orden no solo tiene creencias y deseos, también se atribuyen deseos y creencias a sí mismos y lo hacen a otros... es un sistema recursivo" (p. 38). En este orden de ideas, el humor involucraría no solo la comprensión de las acciones de los personajes por parte del niño, sino también, la comprensión de estados mentales por los cuales se originan esas acciones, esto lo hace una herramienta ideal para capturar la actividad mental del niño.

En cuanto a lo metodológico, el uso de métodos alternativos que intentan seguir detalladamente el proceso del niño en la comprensión de los estados mentales de otros, diferente a las falsas creencias, ha ido ganando terreno. Una de esas alternativas la plantea el método microgenético (Amsterlaw \& Wellman, 2006; Flynn, 2006; Flynn, O'Malley \& Wood, 2004; Puche-Navarro, 2004; Puche-Navarro \& Ossa, 2006). Estos abordajes se relacionan con una tendencia actual en psicología del desarrollo que intenta avanzar en una mirada más detallada y parsimoniosa de cara a lograr una comprensión más precisa de los cambios subyacentes al desarrollo infantil (Adolph, Robinson, Young \& Gill-Alvarez, 2008; Puche-Navarro \& Ossa, 2006; Puche-Navarro, Ossa \& Guevara 2006; Siegler, 2007; Siegler \& Svetina, 2002, 2006; Spencer \& Perone, 2008).

En la línea de estos planteamientos, el presente estudio se propone enfocar la comprensión de los deseos de los niños a través de dos tipos de situaciones experimentales. Por una parte con la 
réplica de los estudios de preferencias y por otra parte, con la utilización de situaciones con humor gráfico y específicamente de chistes mentalistas. La conjetura es que ambas situaciones se apoyan en procesos inferenciales que les son subyacentes, y se puede lograr entonces evidencia empírica sobre la complejidad de la actividad representacional en edades tempranas.

\section{Método}

\section{Diseño}

Esta investigación utilizó el método microgenético con un seguimiento de 38 sujetos a lo largo de 10 sesiones, con intervalos de 8 días entre sesión, para un total de dos meses y medio de aplicaciones. En cada sesión se usaron dos versiones de cada una de las tareas (humor y preferencias) y se repitieron dos veces a todos los participantes. Se contrabalanceó el orden de aplicación a través de las sesiones.

\section{Participantes}

38 niños de 2.8 a 3 años ( $47 \%$ niñas y $53 \%$ niños) pertenecientes a 4 preescolares de la ciudad de Palmira/CO, de un estrato socioeconómico 3 y 4 (medio) según registros del DANE. La intervención con los niños se llevó a cabo con previa autorización de los padres quienes firmaron un consentimiento informado y su participación en el estudio fue voluntaria.

\section{Materiales}

Se usaron dos situaciones experimentales, una de humor gráfico y una de las empleadas tradicionalmente en TdM para explorar comprensión de deseos (preferencias).
Situación de humor. Se emplea una situación de resolución de problemas de humor gráfico inspirada en las de Puche-Navarro (2004), la cual previamente se ha seleccionado por medio de un estudio piloto. La tarea es compuesta por tres imágenes impresas en cartulina full color de $20 \times 20 \mathrm{~cm} \mathrm{c/}$ una, las cuales se diferencian según su contenido semiótico: una congruente, que no plantea ningún elemento humorístico; una mentalista que proporciona información acerca del deseo o creencia del personaje (incongruencia mentalista); y una neutra, que aunque no pertenece al sistema, no causa conflicto, por tanto no es chistosa (figura 1). Se crearon cuatro versiones de la misma tarea y en todas ellas el protagonista es un animal, el cual muestra su deseo por medio de una situación incongruente representada en una burbuja. Las versiones presentadas a los niños fueron: el domador y el mico del circo (un mico haciendo malabares que se imagina a su entrenador en una jaula entregándole un banano), el pez y el entrenador (el pez se imagina tirando peces al entrenador mientras este brinca como un delfín), el pez y la paloma (Figura 1), el pescador pescado (un pez que se imagina en la barca pescando al pescador). A todas estas versiones subyace la misma organización semiótica y se crearon para evitar el efecto de repetición en los resultados de los niños. Se formulan a los niños tres preguntas que serán explicadas en el procedimiento.

Análisis semiológico. El análisis semiológico representa una forma de acceder a las demandas cognitivas que implican los chistes. Además, provee información sobre las diversas relaciones de los elementos contenidos en la imagen y permite identificar las violaciones de segundo orden, sobre las cuales se construyen las imágenes incongruentes. La Tabla 1 (adaptación de Puche-Navarro, 2004) muestra el análisis semiológico de una de las versiones de los chistes empleados en el presente estudio, el del pez y la paloma (Figura 1). 
Tabla 1.

Análisis semiológico de una de las versiones de la tarea

\begin{tabular}{|c|c|c|}
\hline Descripción & Análisis & Exigencia Cognitiva \\
\hline $\begin{array}{l}\text { En esta imagen } \\
\text { un pez observa } \\
\text { por la ventana } \\
\text { desde su pece- } \\
\text { ra a una palo- } \\
\text { ma que vuela } \\
\text { libre. A su vez, } \\
\text { se imagina que } \\
\text { él puede volar } \\
\text { como ella, pero } \\
\text { con un globo su- } \\
\text { jetado a él. }\end{array}$ & $\begin{array}{l}\text { Violación de segundo orden: En esta } \\
\text { imagen se hace una atribución al es- } \\
\text { tado mental del pez, quien desea vo- } \\
\text { lar como lo hace la paloma, pero al no } \\
\text { tener alas se imagina con una bomba } \\
\text { atada a su cuerpo para poder hacerlo. } \\
\text { Aquí se evidencian tres transgresiones, } \\
\text { (1) el pez realiza una actividad humana, } \\
\text { pensar, desear; (2) el pez requiere de } \\
\text { un elemento de creación humana para } \\
\text { poder volar, un globo y (3) los peces no } \\
\text { pueden salir del agua, si no viven en el } \\
\text { agua mueren. }\end{array}$ & $\begin{array}{l}\text { El sujeto debe identificar todos los elementos invo- } \\
\text { lucrados en la situación: el pez, la paloma, el globo, } \\
\text { el acto de volar, de pensar. Recupera la violación } \\
\text { de la incongruencia bajo la función que puede tener } \\
\text { esta y establece comparaciones con la representa- } \\
\text { ción mental del objeto propuesto y la imagen del } \\
\text { chiste: identifica el estado mental del pez quien tie- } \\
\text { ne un deseo, logra inferir que a partir de situacio- } \\
\text { nes convencionales en las cuales una paloma vuela } \\
\text { se genera una situación humorística en la cual el } \\
\text { personaje, en este caso el pez, desea volar como la } \\
\text { paloma para ser libre. Esto se encuentra atribuido } \\
\text { a un funcionamiento analítico y de tipo inferencial. }\end{array}$ \\
\hline
\end{tabular}

Figura 1. Versión 1. Chiste del pez y la paloma.

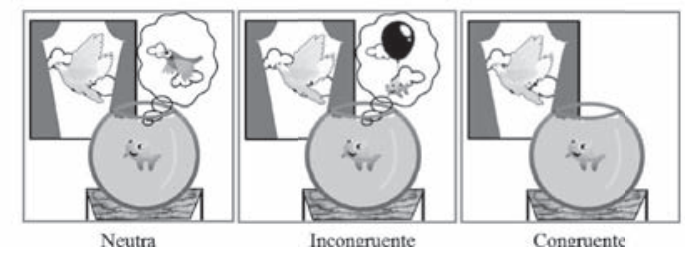

Situación de preferencias. El escenario consiste en un juego con imágenes en el que el niño actúa como ayudante de un duende quien está buscando el regalo adecuado para cada uno de tres niños. Para ello cuenta con tres pistas de cada niño que corresponden a diferentes categorías (preferencias de cada personaje): juguete favorito, actividad favorita y sitio favorito. En estas preferencias y en la meta (dar a cada personaje lo que más le gusta) se encuentran operacionalizados los deseos. En total se usan cuatro versiones de la misma tarea. La consigna de la tarea es la siguiente: "este es el duende ayudante de Papá Noel, es un duende muy despistado y confundió los regalos que debía entregar a tres niños, tú debes ayudarlo a escoger cuál regalo sería el de cada niño, teniendo en cuenta que papá Noel les compró el regalo que más les gusta a cada uno, para eso tienes tres pistas por cada niño que les ayudan a descubrir el dueño de cada regalo". Posteriormente, el participante debe ubicar el regalo de cada uno de los personajes (imagen de 10 $x 10 \mathrm{~cm}$ ) en una plantilla de $21 \times 43 \mathrm{~cm}$ que contiene los dibujos de cada niño en el encabezado y los espacios en blanco para ubicar las pistas y los regalos, tal y como lo muestra la Figura 2.

Figura 2. Plantilla utilizada en la tarea de preferencias

\begin{tabular}{lll}
\hline MATEO & LAURA & DANIEL \\
\hline Lugar para ubi- & Lugar para ubi- & Lugar para ubi- \\
car evidencia 1 & car evidencia 1 & car evidencia 1 \\
Juguete & $\begin{array}{l}\text { Juguete } \\
\text { favorito }\end{array}$ & $\begin{array}{l}\text { Juguete } \\
\text { favorito }\end{array}$ \\
favorito & Lugar para ubi- & Lugar para ubi- \\
Lugar para ubi- & Lugar \\
car evidencia 2 & car evidencia 2 & car evidencia 2 \\
Actividad & Actividad & Actividad \\
favorita & favorita & favorita \\
Lugar para ubi- & Lugar para ubi- & Lugar para ubi- \\
car evidencia 3 & car evidencia 3 & car evidencia 3 \\
Sitio favorito & Sitio favorito & Sitio favorito \\
ESPACIO PARA & ESPACIO PARA & ESPACIO PARA \\
UBICACIÓN DEL & UBICACIÓN DEL & UBICACIÓN DEL \\
REGALO & REGALO & REGALO \\
\hline
\end{tabular}

\section{Procedimiento}

Las dos tareas se aplicaron en la misma sesión. Se trabajó con cada uno de los niños fuera del salón de clases individualmente. Se usó una mesa de las que generalmente se emplean en los pre-escolares. 
Tarea de humor. En esta tarea se realizó una familiarización en la cual se habló con el niño acerca de ¿qué es chistoso? ¿Qué programas de televisión ha visto chistosos? ¿Qué lo hace reír más? Cuando se terminó de conversar con el niño sobre este tema, se organizaron las tres tarjetas (solo una versión) sobre la mesa, una seguida de otra y se le pidió al niño identificar cada uno de los elementos en las imágenes. Cuando el experimentador se había asegurado de que el niño comprendió todos los elementos de las imágenes se realizaron las preguntas correspondientes a la tarea en el siguiente orden: 1) ¿cuál de estas imágenes es la más chistosa? 2) Si el pez (o animal protagonista de la lámina) nos quiere decir qué es lo que quiere ¿con cuál imagen podría hacerlo? y 3) en esta imagen (la incongruente) ¿qué es lo chistoso? La Figura 1 muestra un ejemplo de una de las versiones de la tarea. Las respuestas de los niños se registraron en una rejilla y adicionalmente se realizó una grabación de voz en un teléfono celular para asegurarnos de la información proporcionada.

Tarea de preferencias. En esta tarea se le pidió al niño identificar cada una de las láminas, si el niño no las comprendía el experimentador se las describió hasta asegurarse que estaban claras para el participante. Después de esta breve familiarización se le dijo la consigna al niño y se le entregaron las imágenes con los regalos para ubicarlos en cada una de las tres columnas. Siempre quedó un regalo sobrando que correspondió a una categoría neutra. Las elecciones de los niños eran registradas en una rejilla y adicionalmente se realizó una grabación de voz.

\section{Análisis de datos}

Se conduj eron dos tipos de análisis: uno intragrupo y uno intrasujeto. En el intragrupo se compararon los desempeños de los participantes en las dos tareas, se realizó un análisis de frecuencias y se corrió una prueba de Kruskal-Wallis $\mathrm{H}$. El análisis intrasujeto se realizó para comparar las tendencias en las movilizaciones de los desempeños de los participantes en las 10 sesiones, para ello se utilizó la técnica de movimientos mínimos y máximos. Esta es una técnica de los sistemas dinámi$\cos$ no lineales que permite ilustrar desempeños en un número repetido de observaciones y arroja tendencias en las movilizaciones de los participantes a lo largo de las sesiones de aplicación.

Criterios de comprensión y definición de los funcionamientos mentales subyacentes a las dos tareas. Tanto en la situación de humor como en la de deseos, el niño debe hacer una predicción de los estados mentales de otro. Como se sabe, buena parte de esa predicción supone un sistema inferencial relativamente sólido. Ambas tareas tienen como objetivo central la detección de la comprensión de los deseos por parte de los niños. La predicción del estado mental del deseo del personaje, en la tarea humor se identifica en la proyección que se operacionaliza en la burbuja. El niño lee esa burbuja como lo que el sujeto 'desea'. Por su parte, la predicción del estado mental en la tarea de preferencias se hace a partir de la recuperación de indicios que dan cuenta de los gustos o preferencias de los personajes. A partir de esta información el niño debe elegir un regalo que resultó deseado para cada personaje.

En el caso de la situación de humor gráfico, el niño debe identificar los elementos de la situación y recuperar el elemento incongruente, el niño identifica la violación de lo real e infiere la relación de esta violación con los estados mentales del personaje involucrado en la imagen. El deseo juega un papel crucial en la medida que es el motor de las acciones del personaje que aún no han sido llevadas a cabo, pero que por medio de lo imaginativo se pueden desarrollar y así darle otro nivel de satisfacción. En la situación de preferencias, el objeto de deseo se infiere de una categoría en la cual se encuentran caracterizadas las preferencias de los personajes, el niño debe identificar esta categoría, relacionarla con los elementos disponibles para regalar y realizar la elección. De igual forma, se puede afirmar que ambas tareas permiten explorar la inferencia de los deseos, sin embargo, lo hacen tomando evidencia distinta. En ambos casos se requiere un funcionamiento inferencial y el diseño de las dos tareas está construido sobre tres preguntas centrales: 1) ¿se trata de los mismos funcionamientos inferenciales? sino no es el caso 2) ¿cuál es la di- 
ferencia? o 3) ¿puede esta diferencia deberse a un problema metodológico?

El orden de la exposición siguiente se elabora sobre esas preguntas. Ahora bien, en el análisis de tareas se identificó que en lo metodológico las evidencias proporcionadas al niño son distintas entre una tarea y otra. En la situación de humor la evidencia es la proyección en la burbuja, aunque se podría decir que en la imagen ya se encuentra explícito el deseo. Por ejemplo, un pez en su acuario mira una paloma volar y se imagina él mismo volando con un globo atado a su cuerpo. En el caso de la tarea de TdM la información sobre el deseo del sujeto se ofrece en los indicios sobre el gusto o preferencia de los personajes por un tipo de juguete, actividad o sitio en particular. Por ejemplo, al niño se le dice "esta es Laura, su juguete favorito son los patines, su actividad favorita es montar bicicleta y su sitio favorito es el parque" y así se le da la misma información de tres personajes. Así pues, se parte de dos tipos de evidencia para llegar al mismo punto: vehicular o identificar la predicción del deseo de los personajes.

Tabla 2.

Definición de los funcionamientos inferenciales subyacentes a las dos tareas

Nivel,
puntajes y acciones

Transicional (puntajes 4, 5 y 6 )

En este nivel el sujeto comienza a establecer relaciones entre los elementos fundamentales del chiste y encuentra el camino intermedio entre una definición explícita de todas las relaciones y la descripción de los elementos implicados en el chiste.
Explícito (puntajes 7, 8 y 9)

Las acciones de este nivel están caracterizadas por una identificación explícita de las relaciones que vinculan los eventos del chiste con el estado mental del personaje. En la mayoría de los casos el niño hace alusión a los estados mentales del protagonista.

\section{Funcionamiento - humor \\ Funcionamiento - deseos}

El funcionamiento que subyace a esta elección se ubica en un nivel descriptivo muy inicial, que responde principalmente a características dentro de las relaciones convencionales del objeto sin mostrar acceso a las relaciones planteadas por la incongruencia que conforman el tipo de humor gráfico referido y significan al chiste gráfico.

El funcionamiento que subyace a esta elección comienza a incorporar elementos de la realidad subvertida en el chiste. Sin embargo, el niño aún se encuentra sujeto a una lectura literal de la imagen.

Este funcionamiento se basa en la inferencia propiamente dicha. El niño toma la evidencia presente en la proyección (burbuja) para identificar claramente el deseo involucrado.
El funcionamiento que subyace a esta elección se ubica en un nivel descriptivo muy inicial, que responde principalmente a los indicios que se han ofrecido en la imagen. Las relaciones entre la preferencia del personaje y el regalo que debe asignar el niño no se encuentran presentes. El niño se orienta básicamente por su propio deseo.

Aunque la relación entre la evidencia presentada en la preferencia parece ser comprendida por las elecciones realizadas, no es claro que el niño infiera el deseo de los personajes. Se podría decir que aparecen los primeros esbozos de una inferencia que no logra concretarse.

Este funcionamiento se basa en la inferencia. El niño debe operar con información conocida (preferencias) que corresponde a los deseos en un área específica de cada personaje, un juguete, sitio favorito y actividad favorita.

De esta forma, el niño debe realizar una inferencia sobre el regalo deseado por cada personaje basado en las evidencias.

Para llegar a determinar el regalo de cada personaje, debe tomar cada evidencia y vincularla a una categoría general que le permite llegar a revisar cada uno de los objetos e inferir a qué categoría corresponde cada uno. 
A partir de estas consideraciones se construye una escala de criterios de puntuación (para las dos tareas) que asigna un puntaje de 1 a 9 de acuerdo con la justificación y con la elección proporcionada por el niño al enfrentarse a cada una de las versiones de la tarea. Después de haber definido los nueve puntajes se agrupan en uno de tres niveles: implícito, transicional y resolutorio. El implícito comprende los puntajes 1, 2 y 3; el transicional contiene los puntajes 4, 5 y 6; y el explícito, los puntajes 7, 8 y 9. Cada uno de estos niveles se operacionaliza en el análisis de tareas de la Tabla 2.

\section{Resultados}

A continuación se presentan los resultados globales de 38 participantes, promediando los desempeños en las 10 sesiones de aplicación, frente a las dos tareas, humor y preferencias. Los resultados distribuidos en los puntajes de la escala (1 a 9) se agrupan en tres niveles implícito, transicional y explícito (Tabla 2).

Frecuencia de participantes por nivel en las dos tareas. La figura 2 condensa la frecuencia de participantes según los niveles tomando todas las aplicaciones de manera conjunta. Veamos la comparación de los desempeños frente a las dos situaciones: de humor y de preferencias (Figura 3).

Figura 3. Porcentaje de participantes en los tres niveles en todas las sesiones de aplicación

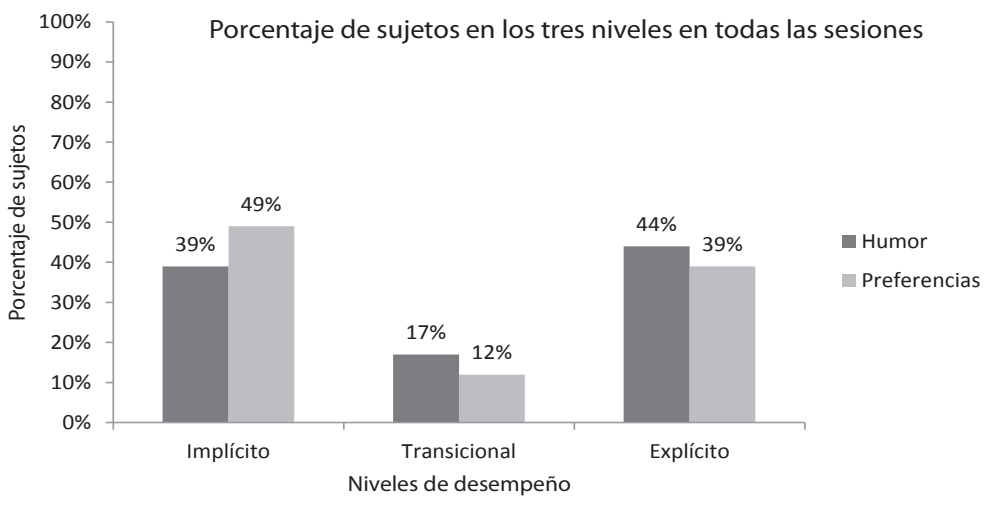

Como se observa en la Figura 3, es marcada la semejanza de los desempeños ante las dos situaciones. Respecto de la distribución de participantes por niveles, el nivel transicional presenta el menor número de participantes mientras que los niveles implícito y explícito tienen un número de participantes parecido. La homogeneidad de los desempeños de los participantes ante las dos situaciones resulta entonces el elemento de mayor relieve, y con base en esos datos se puede afirmar que las dos tareas parecen responder a exigencias similares, como lo mostraba el análisis de tareas.

La prueba no paramétrica de Kruskal-Wallis $\mathrm{H}$ comparó los intentos entre cada sesión en las dos tareas para controlar el efecto de repetición de los participantes y mostró que no existen diferencias significativas entre los intentos de una tarea y otra.

Esa similitud entre los desempeños parecería indicar que, la manera como el niño entiende ayudar al duende despistado de la situación de preferencias es muy similar a la manera como entiende los deseos del pez frente a la paloma, a pesar de que estas situaciones de humor resultan a primera vista un tanto más complejas. A partir de esas semejanzas es factible considerar que la tarea del humor y la de preferencias de deseos involucran funcionamientos cognitivos de la misma naturaleza, y que en este caso parece ser los funcionamientos inferenciales. 
Movilizaciones del grupo. Además de las similitudes de los desempeños entre las dos situaciones los resultados mostraron una gran movilidad a lo largo de las distintas aplicaciones. Como se recordará el diseño en series de tiempo, toma desempeños de los participantes a partir de 10 aplicaciones con intervalos de 8 días a lo largo de dos meses y medio. Los seguimientos a lo largo de las 10 aplicaciones se llevaron a cabo con la técnica de movimientos de Mínimos y Máximos que como se describió en los aspectos metodológicos es una técnica que permite graficar los itinerarios de los participantes. Se identificaron tres tipos de movilizaciones: un tipo de movilización ascendente, cuando entre la aplicación 1 y la 10 la tendencia de los participantes marcó un movimiento ascendente en las puntuaciones. En segundo lugar, un tipo de movilización estable, cuando a lo largo de las 10 sesiones se encontró una recurrencia del mismo nivel de desempeño. Finalmente, un tipo de movilización descendente, cuando los puntajes a lo largo de las 10 aplicaciones tendieron a disminuir. La prueba de K-means confirmó tres conglomerados de participantes que correspondían precisamente a las tres tendencias en las tres movilizaciones antes descritas. Las gráficas permitieron agrupar tres tipos de tendencias de los participantes para cada una de las tareas: ascienden, estables y descienden (Figura 4).

Figura 4. Comparación entre tendencias de movilizaciones en las dos tareas.

\section{Tendencias de movilizaciones en las dos tareas}

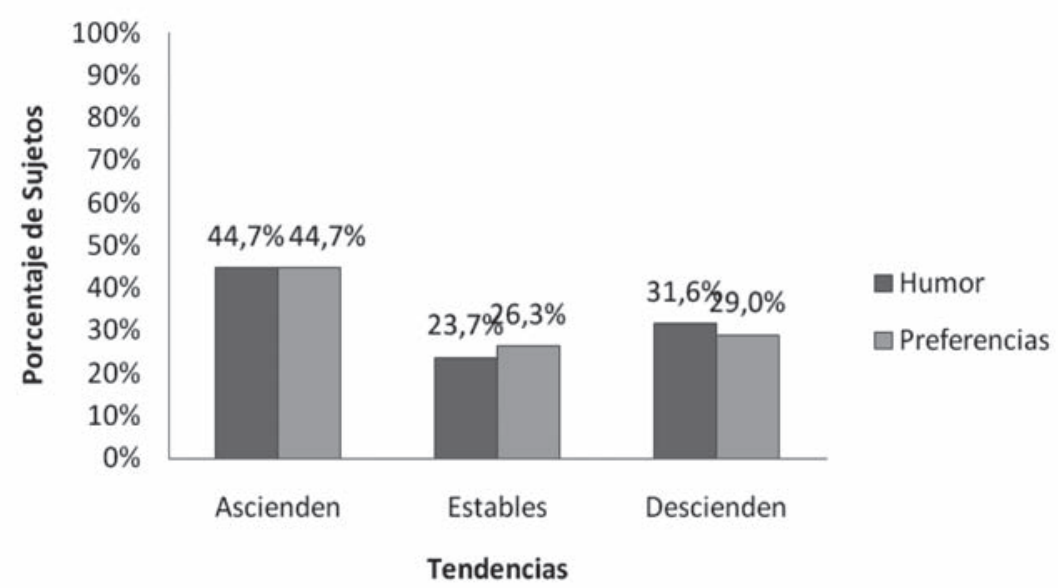

Aunque la semejanza de los participantes en las dos situaciones fue un dato importante y sorprendente, lo fue doblemente el encontrar que las movilizaciones de las que da cuenta la gráfica de mínimos y máximos a lo largo de los dos meses de seguimiento, son prácticamente idénticas. En otras palabras, casi el mismo número de participantes ante las dos situaciones arroja el mismo tipo de movilizaciones. El mayor porcentaje de participantes, un $44,7 \%$ (17/38), presenta movilizaciones ascendentes, un número reducido de participantes presenta desempeños estables y lo más sorprendente es registrar movilizaciones descendentes en un $30 \%$ de los participantes. La cuestión entonces es lo que quiere decir la semejanza de estas movilizaciones ante las dos situaciones.

\section{A manera de balance}

Como se observa en la Figura 3 las tendencias de las movilizaciones en los desempeños de los participantes para las dos tareas fueron extraordinariamente similares, los datos frente a una y otra situación son muy homogéneos y corroboran la homogeneidad de los resultados en los tres ni- 
veles de comprensión. La semejanza de los resultados en las movilizaciones confirma que ambas situaciones son equiparables entre sí. Pero ¿qué quiere decir que las movilizaciones de los desempeños de los participantes sean tan coincidentes? Esa similitud puede estar ligada a que se trata de los mismos funcionamientos inferenciales que les subyace. En cierta forma las tendencias en las movilizaciones reflejan más coincidencia que los propios desempeños tomados de manera aislada, lo que puede ser significativo, dado que dan cuenta de que la naturaleza de los funcionamientos inferenciales explorados por diferentes vías parece ser definitivamente la misma.

\section{Elementos para una discusión}

El objetivo de este estudio era documentar la comprensión de los deseos en los niños entre 2.8 y 3 años por medio de dos situaciones experimentales diferenciadas $y$, por otra parte, aportar información sobre las situaciones de humor como escenarios adecuados para el estudio de la teoría de la mente. Se parte de los estudios tradicionales sobre estados mentales enmarcados en metodologías convencionales (tareas de preferencias), y como alternativa a estos estudios y métodos se proponía una situación de resolución de problemas de humor gráfico, así como el uso del método microgenético y las series de tiempo. Para efectos del presente artículo se trabajaron los datos en su conjunto.

En el caso de la tarea de preferencias se retomó como plataforma las situaciones de resolución de problemas clásicas de la teoría de la mente (Cassidy et ál., 2005; Moore et ál., 1995) y se modificó para usarla como una situación abierta con diferentes niveles de resolución. Por su parte, en lo que concierne a la situación de humor, se seleccionaron distintas versiones de las situaciones de chistes mentalistas trabajadas en estudios previos (Puche-Navarro, 2004, 2009). Inicialmente, el análisis de tareas y los desempeños encontraron y mostraron tres niveles de funcionamiento: el nivel implícito, el transicional y el explícito. Esos resultados confirmaron la similitud entre la plataforma sobre la cual están construidas las situacio- nes de humor y de preferencias. La idea de fondo es que los mismos funcionamientos inferenciales subyacen a las dos situaciones experimentales.

Los resultados encontrados demostraron que la tarea de humor y la de preferencias pueden ser equiparables entre sí, situando las tareas de humor gráfico, específicamente los chistes mentalistas, como un escenario ideal para el estudio de los deseos en el niño. Esto quiere decir que los resultados señalan que se puede validar el uso del humor gráfico como instrumento en el estudio de la teoría de la mente, lo que no resulta un dato desdeñable.

Ahora bien, más allá de la validación del abordaje de los estados mentales por vía del humor gráfico, la potencia que tienen los chistes mentalistas en cuanto a lo que ellos nos informan de la TdM, resulta un dato fenomenal. En efecto, los resultados obtenidos permiten confirmar que la comprensión de deseos a partir del tipo de humor gráfico mentalista aparece muy temprano. Este dato llama la atención toda vez que el humor mentalista agencia un funcionamiento inferencial exigente y sofisticado. La niña de tres años se muestra capaz de entender, en el caso del chiste del pez y la paloma, sistemas de segundo orden, como los que formula Dennett para la TdM. En otras palabras, en un primer nivel debe identificar el deseo del pez proyectado sobre la capacidad de volar de la paloma, y en un segundo nivel debe compensar la incapacidad de volar del pez con la utilización de un globo que lo eleve por los aires, para volar como la paloma. Esta misma doble exigencia o sistema de segundo orden se encuentra en la naturaleza de la incongruencia de los otros chistes, por ejemplo, frente al chiste del mico malabarista del circo, la niña debe entender la incongruencia en ese chiste, a saber, la inversión de papeles entre el mico con su domador, que plantea una relación de segundo orden (el mico en el lugar del domador y el domador en el lugar del mico) que es lo que le da sentido a la situación del chiste. El niño o la misma niña de la que veníamos hablando debe comprender que en la jaula (la casa del mico) deberá permanecer el domador, como un deseo inconfesado del mico. 
La capacidad de captar el sentido del humor de estos chistes mentalistas da cuenta de las relaciones inversas allí implicadas y eso es lo que entendemos cuando se dice que la niña o niño que entiende el humor mentalista trabaja bajo sistemas de segundo orden. Lo sorprendente es que esas relaciones resultan tan intrincadas como precoces al aparecer en una edad tan temprana. En tanto que sistemas de segundo orden, ellas son cercanas y muy similares a las requeridas por la $\mathrm{TdM}$. Ambos escenarios exigen identificar, y un desdoblamiento cercano al pensamiento recursivo, es decir, ir más allá que la lectura literal del hecho. La comprensión de los deseos que no son visibles se vehiculan de maneras diversas. No en balde algunos autores lo afirman muy explícitamente "Ios niños de $2 \frac{1}{2}$ años conocen muchos caminos en los cuales un estado mental puede ser el objeto intencional de otro estado mental -el niño conoce caminos en los cuales un estado mental puede llevar o ayudar a causar otro estado mental-" (Wellman, Phillips \& Rodríguez, 2000, p. 909). En síntesis, la infancia parece dotada para captar una diversidad de modalidades de pensamiento, muchas de ellas bastante sutiles.

La cuestión es que la comprensión de ese tipo de relaciones, en tanto sistemas de segundo orden, son relativos a las TdM, en la medida en que suponen la capacidad mental de los niños de tres años de hacer especies de bucles mentales, que es lo esperado en las situaciones TdM. En efecto, algunos autores hablan de esta recursividad mental, aunque no necesariamente de $\mathrm{TdM}$, es el caso de Bateson (1972) y de Perinat (1995). La recursividad mental es la capacidad de remitir a otro componente y a otro significado que carecería de evidencias literales, y que en cambio utiliza la información implícita en los estados mentales. Para dicha recursividad se realizan saltos mentales sobre las propias evidencias, avanzando muy lejos en la significación y abriendo un panorama enriquecido. Lo que estos datos mostrarían sería que esos funcionamientos parecen formar parte de aquellos de que dispone el niño desde muy temprano. Por lo demás, esos funcionamientos están en el centro mismo de los funcionamientos que requiere la TdM, y nuevamente la cita que se acaba de hacer de Wellman y colaboradores va en esta misma dirección.

La otra cuestión que llama la atención es ¿por qué el componente humorístico encerrado en la incongruencia facilita esa temprana comprensión de los estados mentales? La respuesta se puede orientar hacia la construcción de la tarea en sí misma. En primer lugar, las investigaciones sobre comprensión de deseos se han encargado de mostrar la aparición de la comprensión de este estado mental por parte de los niños desde aproximadamente los dos años y medio. Sin embargo, adicionalmente a la documentación de la emergencia de la comprensión de los deseos, los investigadores coinciden con el planteamiento de que este es un estado mental simple y que antecede a la TdM (Cassidy et ál., 2005; Rackoczy et ál., 2007; Repacholi \& Gopnik, 1998; Wellman, 1990; entre otros). De esta forma, las tareas diseñadas son situaciones cerradas que solo implican que el niño haga una elección correcta o incorrecta, las respuestas solicitadas al niño son sí o no. Los escenarios sobre los cuales se construyen estas situaciones carecen de posibilidades para que el niño muestre un funcionamiento mental complejo y sofisticado. Ahora bien, en el caso de las situaciones de humor gráfico se parte de que el niño piensa naturalmente de manera sofisticada y las tareas están construidas sobre bases cercanas al mundo que lo rodea. La contextualización de las tareas dentro de un escenario que le genera sorpresa y a su vez goce y disfrute al niño, permiten que las relaciones que se bosquejan en los elementos del chiste puedan ser recuperadas, representadas, y reconstruidas fácilmente tomando distancia del sentido literal de la imagen para evidenciar el deseo del personaj e y así dar cuenta la comprensión de estados mentales. Es claro que la comprensión de los deseos implica operaciones inferenciales muy complejas que confirman lo exigente de la actividad representacional del sujeto y las situaciones de humor gráfico posibilitan descubrir esa complejidad en su razonamiento.

Finalmente, la presente investigación apoya la idea de la construcción de nuevos escenarios que faciliten la comprensión de estados mentales por parte del niño, ya sean creencias, deseos o inten- 
ciones en entornos relacionados con su cotidianidad. Se resalta la importancia de concebir al niño como suj eto que transforma conocimiento y logra tomar distancia de lo literal para analizar de manera muy fina eventos que rompen con lo convencional. La metodología se orientó al seguimiento detallado de los desempeños de los niños y a permitirles poner en evidencia hipótesis e inferencias sobre los elementos incongruentes y de preferencias que dan cuenta de los estados mentales de otros. Se pudieron identificar las regularidades de ese pensamiento por medio de tres tendencias de movilizaciones. De esta forma, se deja la puerta abierta a futuros trabajos que se interesan por la complejidad de la mente infantil y en especial por la comprensión que los niños tienen de la mente de otros. El humor gráfico se confirma como un escenario útil para explorar la actividad mental del niño. Se invita a los investigadores de la TdM a descubrir, por otras vías, las formas de razonamiento de los niños y a diseñar escenarios que permitan evidenciar en la actividad del niño la comprensión de la mente de los otros.

\section{Referencias}

Adolph, K., Robinson, S., Young, J. \& Gill-Alvarez, F. (2008). What Is the Shape of Developmental Change? Psychological Review, 115(3), 527-543.

Amsterlaw, J. \& Wellman, H. (2006). Theories of mind in transition: A microgenetic study of the development of false belief understanding. Journal of Cognition and Development, 7, 139-172.

Arbeláez, C., Salgado-Montejo, A. \& Velasco, A. (2010). El juego de ficción y la teoría de la mente en niños con dificultades sociales. Revista Diversitas: Perspectivas en Psicología, $6(1), 13-25$.

Astington, J. W. (1998). El descubrimiento infantil de la mente. (T. del Amo, Trad.) Madrid: Morata. (Trabajo original publicado en 1993).

Astington, J. W., Harris, P. \& Olson, D. (1988). (Eds). Developing Theories of Mind. New York, EE. UU: Cambridge University Press.
Astington, J. W. \& Barriault, T. (2001). Children's theory of mind: How young children come to understand that people have thoughts and feelings. Infants and Young Children, 13(3), 1-12.

Bartsch, K. \& Wellman, H. (1989). Young children's attribution of action to beliefs and desires. Child Development, 60, 946-964.

Bariaud, F. (1983). La genèse de I' humour chez I'enfant [The emergence of humor in young children]. Paris: PUF.

Baron-Cohen, S., Leslie, A. \& Frith, U. (1985). Does the autistic children have a theory of mind? Cognition, 21, 37-46.

Bartsch, K. \& Wellman, H. (1995). Children talk about the mind. N.Y: Oxford University Press.

Bateson, G. (1972). Pasos hacia una ecología de la mente: colección de ensayos en antropología, psiquiatría, evolución y epistemología. Northvale, N. J.: J ason Aronson Inc.

Benavides, J. (2010). ¿Engañan los niños antes de los 3 años? Una perspectiva desde el desarrollo de la teoría de la mente. Psicología desde el Caribe, 26, 142-155.

Benavides, J. \& Roncancio, M. (2009). Conceptos de desarrollo en estudios sobre teoría de la mente en las últimas tres décadas. Avances en Psicología Latinoamericana, 27(2), 297-310.

Benavides, J. \& Roncancio, M. (2011). Tres debates fundamentales en el campo de la Teoría de la Mente: aspectos teóricos y metodológicos. Acta Colombiana de Psicología, 14(1), 109-118.

Bloom, P. \& German, T.P. (2000). Two reasons to abandon the false belief task as a test of Theory of Mind. Cognition, 77, 25-31.

Brandone, A. \& Wellman, H. M. (2009). You can't always get what you want: Infants understand failed goal-directed actions. Psychological Science, 20, 85-91. 
Cadinu, M.R. \& Kiesner, J. (2000). Children's development of a theory of mind. European Journal of Psychology of Education, 15, 93-111.

Cassidy, K. W. (1998). Preschoolers' use of desires to solve theory of mind problems in a pretense context. Developmental Psychology, 34(3), 503-511.

Cassidy, K.; Cosetti, M.; Jones, R.; Kelton, E.; Meier, V.; Richman, L. \& Stanhaus, H. (2005). Preschool children's understanding of conflicting desires. Journal of Cognition and Development, 6(3), 427-454.

Combariza, E. \& Puche-Navarro, R. (2009). El uso de la wavelet para el estudio de los funcionamientos inferenciales en niños pequeños. En R. Puche (Comp.). ¿Es la mente no lineal? (pp. 111-133). Cali: Artes Gráficas del Valle Editores.

Días, M.G., Roazzi, A., O’Brien, P. \& Harris, P.L. (2005). Logical reasoning and fantasy contexts: Eliminating differences between children with and without experience in school. Interamerican Journal of Psychology, 39, 13-22.

Doherty, M. (2009). Theory of Mind: How children understand other's thoughts and feelings. New York, USA: Psychology Press.

Eskritt, M. \& Lee, K. (2007). Preescoler's use of eye-gaze for "mind reading". In R. Flom, K. Lee \& D. Muir (Eds.). Gaze-Following: Its development and significance (pp. 243-263). Routledge.

Ferres, L. (2003). Children's early theory of mind: Exploring the development of the concept of desire in monolingual Spanish children. Developmental Science, 6(2), 159-165.

Flavell, J. H., Flavell, E. R., Green, F. L. \& Moses, L. J. (1990). Young children's understanding of fact beliefs versus value beliefs. Child Development, 61, 915-928.
Flom, R., Lee, K. \& Muir, D. (2007). Gazefollowing: Its Development and Significance. Erlbaum: Mahwah NJ .

Flynn, E. (2006). A microgenetic investigation of stability and continuity in theory of mind development. British J ournal of Developmental Psychology, 24(3), 631-654.

Flynn, E., O'Malley, C. \& Wood, D. (2004). A longitudinal, microgenetic study of the emergence of false belief understanding and inhibition skills. Developmental Science, 7(1), 103-115.

Gopnik, A. (1993). How we know our minds: The illusion of first - person knowledge of intentionality. Behavioral and Brain Science, 16, 1-14.

Gopnik, A. \& Astington, J. (1988). Children's understanding of representational change and its relation to the understanding of false belief and the appearance-reality distinction. Child Development, 59(1), 26-37.

Hoicka, E. K. \& Gattis, M. (2008). Do the wrong thing: How toddlers tell a joke from a mistake. Cognitive Development, 23, 180-190.

Hughes, C., Lecce, S. \& Wilson, C. (2007). “Do you know what I want?" Preschoolers' talk about desires, thoughts and feelings in their conversations with sibs and friends. Cognition and Emotion, 21(2), 330-350.

Johnson, K. E. \& Mervis, C. B. (1997). First steps in the emergence of verbal humor: A case study. Infant Behavior \& Development, 20, 187-196.

Lee, K., Eskritt, M., Symons, L. \& Muir, D. (1998). Children's Use of Triadic Eye Gaze Information for "Mind Reading". Developmental Psychology, 34(3), 525-539.

Leslie, A. M. \& Polizzi, P. (1998). Inhibitory processing in the false belief task: Two conjectures. Developmental Science, 1, 247-254. 
Leyva, D. (2003). Cambio en la comprensión de deseos conflictivos en niños de 3 años. (Tesis de maestría no publicada). Universidad del Valle.

Liu, D., Meltzoff, A. \& Wellman, H. (2009). Neural correlates of belief- and desire-reasoning. Child Development, 80(4), 1163-1171.

Loizou, E. (2005). Infant Humor: the Theory of the Absurd and the Empowerment theory. International J ournal of Early Years Education, 13(1), 43-53.

Loizou, E. (2006). Young children's explanation of pictorial humor. Early Childhood Education J ournal, 3(6), 425-431.

Lozano, H. \& Puche-Navarro, R. (1998). Paradigma y sintagma: chiste gráfico y tira cómica: un estudio experimental. Infancia y Aprendizaje, 84, 99-11.

McGhee, P. E. (1979). Humour: Its origin and development. San Francisco: W. H. Freeman.

McGhee, P. E. (1988). The contribution of humour to children's social development. Journal of Children in Contemporary Society, 1(2), 119-134.

Montgomery, D., Bach, L. \& Moran, C. (1998). Children's use of looking behavior as a cue to detect another's goal. Child Development, 69, 692-705.

Moore, C., J arrold, C., Russell, J ., Lumb, A. \& Sapp, F. (1995). Conflicting desire and the child's Theory of Mind. Cognitive Development, 10, 467-482.

Perinat, A. (1995). Prolegómenos para una teoría del juego y del símbolo. Cognitiva, 7(2), 185-204.

Puche-Navarro, R. (2004). Graphic jokes and children's mind: An unusual way to approach children's representational activity. Scandinavian Journal of Psychology, 45(5), 343-355.
Puche-Navarro, R. (2009). From implicit to explicit representation in children's response to pictorial humor. International J ournal of Behavioral Development, 33, 543-555.

Puche-Navarro, R. (2012). Modeling iconic literacy: The dynamic models for complex cultural objects. In J. Valsiner (Ed.). The Oxford Handbook of Culture and Psychology. New York: Oxford University Press.

Puche-Navarro, R. \& Lozano, H. (2002). El sentido del humor en el niño pequeño: un estudio empírico del funcionamiento del chiste y la representación en la infancia. Bogotá: Siglo del Hombre Editores.

Puche-Navarro, R. \& Ossa, J. (2006). ¿Qué hay de nuevo en el método microgenético? Más allá de las estrategias y más acá de la del funcionamiento cognitivo del sujeto. Summa Psicológica, 13(2), 117-137.

Puche-Navarro, R., Ossa, J. \& Guevara, M. (2006). La resolución de problemas ¿Una alternativa integradora? Revista Educación y Cultura, 18(46), 167-190.

Rakoczy, H., Warneken, F. \& Tomasello, M. (2007). "This way!", "No! That way!" 3-year olds know that two people can have mutually incompatible desires. Cognitive Development, 22, 47-68.

Reddy, V. (1991). Teasing, joking and mucking about: Playing with others' expectations. In A. Whiten (ed.) Natural Theories of Mind. Oxford: Blackwel.

Reddy, V. (2001). Infant clowning: the interpersonal creation of humour in infancy. Enfance, 3, 247-256.

Reddy, V. (2006). Getting back to the rough ground: deception and social living. Philosophical Transactions of the Royal Society, 10, 1-15.

Reddy, V. (2008). Experiencing others: A secondperson approach to other-awareness. In: $U$. 
Müller, J.I.M. Carpendale, N. Budwing \& B. Sokol (Eds.) Social Life and Social Knowledge (pp. 123-144). New York, EE.UU.: Lawrence Erlbaum Associates.

Reddy, V. \& Morris, P. (2004). Participants don't need theories: Knowing minds in engagement. Theory and Psychology, 14(5), 647-665.

Repacholi, B. M. \& Gopnik, A. (1997). Early reasoning about desires: Evidence from 14- and 18-month olds. Developmental Psychology, $33,12-21$.

Rieffe, C., Meerum Terwogt, M., Koops, W., Stegge, H. \& Oomen, A. (2001). Preschoolers' appreciation of uncommon desires and subsequent emotions. British Journal of Developmental Psychology, 19, 259-274.

Roncancio, M. (2010). Del estudio de las creencias al estudio de los deseos: una revisión de las tendencias metodológicas. Revista Virtual Universidad Católica del Norte [on line] (29). Recuperado el 13 de febrero de 2010 de: http:// revistavirtual.ucn.edu.co/index. php?option=com_content\&task=view \&id $=127$ \&ltemid=1.

Siegler, R. (2007). Cognitive variability. Developmental Science, 10, 104-109.

Siegler, R. \& Svetina, M. (2002). A microgenetic/ cross-sectional study of matrix completion: Comparing short-term and long-term change. Child Development, 73, 793-809.

Siegler, R. \& Svetina, M. (2006). What leads children to adopt new strategies? A microgenetic/cross sectional study of class inclusion. Child Development, 77, 997-1015.

Spencer, S. \& Perone, J. (2008). Defending qualitative change: The view from dynamical systems theory. Child Development, 79(6), 1639-1647.

Strick, M., Holland, R. W., Van Baaren, R. B. \& Van Knippenberg, A. (2009). Finding comfort in a joke: Consolatory effects of humor through cognitive distraction. Emotion, 9, 574-578.

Taylor, M. (1996). A theory of mind perspective on social cognitive development. En R. Gelman \& T. Kit-Fong (Eds.) Perceptual and cognitive development (pp. 283-328). San Diego: Academic Press.

Terwogt, M. \& Rieffe. C. (2004). Deaf Children's Use of Beliefs and Desires in Negotiation. J ournal of Deaf Studies and Deaf Education, 9(1), 27-38.

Wellman, H. (1990). The child's theory of mind. Cambridge MA: MIT Press.

Wellman, H. \& Banerjee, M. (1991). Mind and emotion: Children's understanding of the emotional consequences of beliefs and desires. Journal of Developmental Psychology, 9, 191-214.

Wellman, H. \& Woolley, J. (1990). From simple desires to ordinary beliefs: The early development of everyday psychology. Cognition, $35,245-275$.

Wellman, H., Phillips, A. \& Rodríguez, T. (2000). Young Children's Understanding of Perception, Desire, and Emotion. Child Development, 71(4), 895-912.

Wertz, A. \& German, T. (2007). Belief-desire reasoning in the explanation of behavior: Do actions speak louder than words? Cognition, 105, 184-194.

Wimmer, H. \& Perner, J. (1983). Beliefs about beliefs: Representation and constraining functions of wrong beliefs in young children's understanding of deception. Cognition, 13, 103-128.

Woolley, J. \& Wellman, H. (1993). Origin and truth: Young children's understanding of imaginary mental representations. Child Development, 64, 1-17. 